\title{
Dynamic Friction-Based Force Feedback for Tendon- Sheath Mechanism in NOTES System
}

\author{
T. N. Do, T. Tjahjowidodo, M. W. S. Lau, and S. J. Phee
}

\begin{abstract}
Tendon-sheath mechanism (TSM) is commonly used in flexible endoscopic systems because of its high flexibility, light weight, and easy transmission. Due to the size constraints and sterilization problems, traditional sensors cannot be placed at the tool tips of robotic arms. In addition, nonlinear friction and backlash hysteresis cause many challenges to predict the desired force when the system inside the human's body. It is extremely difficult to provide the force information to haptic devices and subsequently to the users. In this paper, a new scheme of dynamic friction model for a pair of TSMs is proposed to estimate the force at distal end of endoscopic system. In comparison with current approaches in the literature, our model is able to provide continuous force information. The model is able to predict distal force with any sheath shapes. An experimental setup is designed to measure the friction force in the TSM. Finally, the validity of the proposed model approach is confirmed with a good agreement between the estimated values and real experimental data.
\end{abstract}

Index Terms-Dynamic friction, tendon-sheath, haptic feedback, endoscopic systems.

\section{INTRODUCTION}

Flexible endoscopy allows for treating and inspecting gastrointestinal (GI) disorders without leaving any abdominal incision to the human's body [1]-[5]. One of the promising techniques using flexible endoscope is natural orifice transluminal endoscopic surgery (NOTES). It can approach desired targets using flexible endoscope and robotic arms to perform complex surgical task like suturing. It can operate in small and narrow paths, provides high load and more flexibility. A pair of tendon-sheath mechanism (TSM) is often used to actuate the robotic arm. Although the TSM promises beneficial features for transmission, nonlinearities and backlash hysteresis cause loss of tension from the proximal end to distal end of the system. This can be disadvantages in medical applications where traditional sensors like strain gauges cannot be placed at the tool tips of robotic arm due to the size constraint and sterilization problems.

Haptic interface are devices that provide and simulate the sense of touch from the tissues to the surgeon's hand. In medical applications, it is a vital factor because the surgeon is able to feel the differences between soft or hard objects. With the availability of current haptic devices such as CyberForce,

Manuscript received December 9, 2013; revised March 11, 2014.

Thanh Nho Do, Tegoeh Tjahjowidodo, and Soo Jay Phee are with the Division of Mechatronics and Design, School of Mechanical \&Aerospace Engineering, NTU, Singapore (e-mail: thanh4@e.ntu.edu.sg, TTEGOEH@ntu.edu.sg, MSJPHEE@ntu.edu.sg).

M. W. S. Lau is with the Newcastle University, United Kingdom (e-mail: michael.lau@newcastle.ac.uk).
CyberGrasp, Phantom, the capability of enhancing safe surgery and performances for surgical systems are always possible [6]-[7]. It was reported that haptic feedback to the surgeons would be an essential factor for safe surgery [8]-[10]. Without such feedback, surgeons are not able to feel as they have in direct contact with the real tissues. Since sensors cannot be mounted at the distal end, the capability of providing force information to haptic devices and subsequently to surgeons is extremely difficult. Therefore, mathematical model can be considered as potential substitutes. Recently, several researchers have discussed and analyzed the transmission problems of the TSM in various applications. Kaneko et al. [11]-[13] presented the tension transmission of the TSM using Coulomb friction model. Do et al. [14] introduced the displacement transmission for a single TSM. However, no tension analyses have been discussed. Palli et al. [15]-[16], Tian et al. [17], Chen et al.[18] and Low et.al [19] introduced the transmission for a single TSM with the assumption of the same pretension for small elements Agrawal et al. [20]-[21] used a set of partial differential equations to model a single TSM and a pair of TSM in a closed loop approach. These existing approaches only consider the transmission model for the TSM when the configuration is known and the sheath curve angles are available. In addition, the use of Coulomb model causes discontinuity when the system operates at vicinity of zero velocity. Nonlinear friction in a pair of TSM is quite complex. The forces are different when the system is accelerating and decelerating. Current friction models like Dahl, LuGre, Leuven, or GMS [22]-[23] are not able to describe the complete features of nonlinear friction in a pair of TSM since they mainly model the friction force with the same values in acceleration and deceleration using Stribeck curve. Although Do et al. addressed these problems in [24]-[26], they only considered the friction model for a single TSM and the models possess a high number of parameters. It will cost more time to identification and computation. In this paper, a new dynamic friction model for a pair of TSM will be presented with less model parameters in its structure and easy to implement for haptic problems.

It is known that the normalized Bouc-Wen model can describe a wide range of hysteresis phenomena [27]-[28]. It is used in various applications like magnetorheological or piezoelectric systems. Therefore, an asymmetric dynamic friction model using the modified normalized Bouc-Wen model will be developed to capture the nonlinearity characteristics of a pair of TSM. The ultimate goal of this approach is to provide the force information at the distal end of the endoscopic system without using any sensors at the distal end during operations. Having the force information, 
haptic devices can be utilized to provide necessary force to the surgeons. To validate the proposed approach, suitable experiments will be designed and comparisons will be given. The rest of this paper is organized as follow: Section II discusses the transmission characteristics for a pair of TSM. Section III presents a new dynamic friction model for the approach. Section IV introduces the experimental setup and identification algorithm. The comparisons are drawn in Section V. Finally, the conclusion part is depicted in Section VI.

\section{DYNAMIC PROPERTIES FOR A PAIR OF TSM}

A typical Natural Orifice Transluminal Endoscopic Surgery (NOTES) system is illustrated in Fig. 1. It consists of a master console, a slave manipulator, and a motor housing. One of the main tools of NOTES is an endoscope which is a flexible shaft with an articulated bending tip and tool channels to house the robotic arms as well as camera (provide visual feedback to the surgeon). Surgeons perform the surgical tasks using a master console to control the robotic arms inside the patient's body. The robotic arms, which possess multi degrees of freedom (DOFs), are fixed and are carried along within the endoscope to perform demanding surgical procedures such as suturing and cutting. Each degree of freedom of the arm is actuated using a pair of TSM. Triangulation is carried out at the distal end of the endoscope while actuation is externally provided. Due to the size constraints and sterilization, traditional sensors are not suitable to mount at the joints of robotic arms. Therefore, the control of NOTES system is often open loop. Only visual feedback from the camera is provided during the operation.

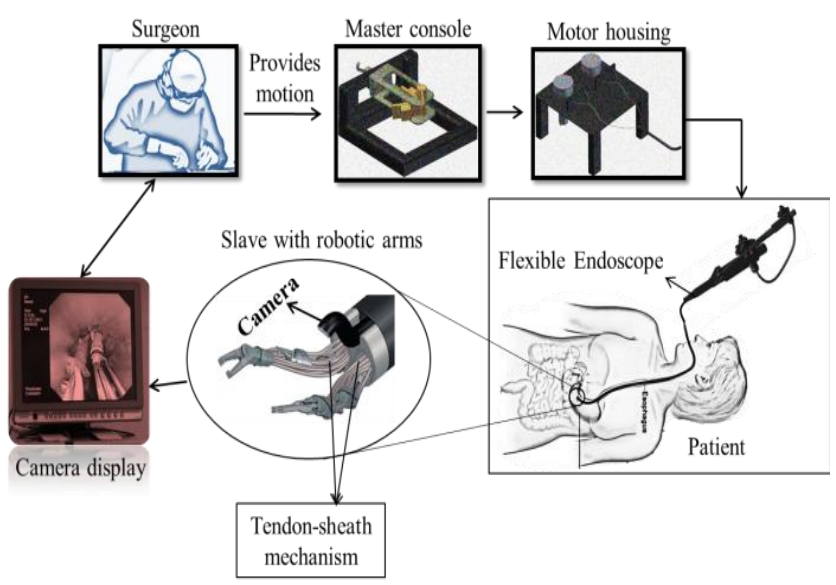

Fig. 1. Overview of a NOTES system.

Fig. 2(a) shows a pair of TSM used in flexible endoscopic systems. $x_{\text {in }}, x_{\text {out }}, \tau_{\text {in }}, \tau_{\text {out }}$ denote the angular displacements and torques at the drive pulley (pulley input) and the follower pulley (pulley output), respectively. $T_{i}(i=1,2,3,4)$ denote the tensions at proximal end and distal end of each of the tendons. The two pulleys have the same radius $R$. The torques are expressed by:

$$
\left\{\begin{array}{l}
\tau_{\text {in }}=R\left(T_{1}-T_{3}\right) \\
\tau_{\text {out }}=R\left(T_{2}-T_{4}\right)
\end{array}\right.
$$

If we denote the friction forces in the Sheath 01 and 02 as $F_{1}$ and $F_{2}$, respectively, then the friction forces in term of tensions at proximal and distal end are given by:

$$
\left\{\begin{array}{l}
F_{1}=T_{1}-T_{2} \\
F_{2}=T_{4}-T_{3}
\end{array}\right.
$$

The total friction forces in the two tendon-sheath mechanisms can be calculated by:

$$
F=\frac{\tau_{\text {in }}-\tau_{\text {out }}}{R}=F_{1}+F_{2}
$$

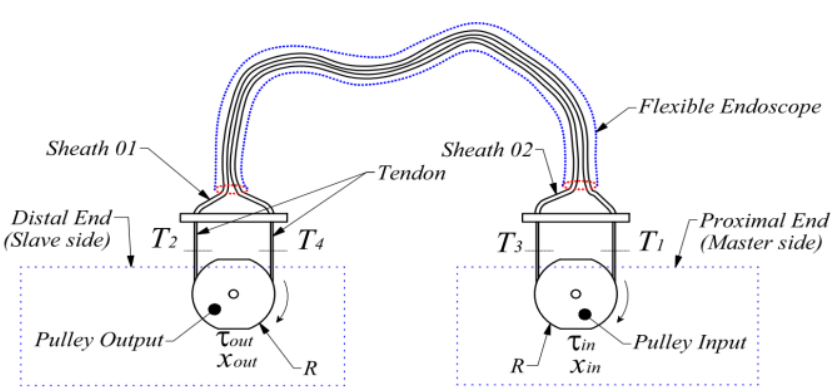

(a)

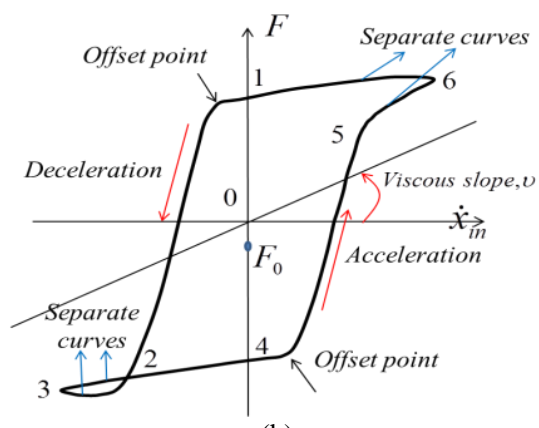

(b)

Fig. 2. A pair of TSM: (a) diagram; (b) transmission property.

The dynamic transmission profiles of a pair of TSM are shown in Fig. 2(b) (the relation between total friction force and relative velocity of proximal end). Suppose that the motion of pulley input firstly rotates in clockwise direction (positive velocity) as shown in Fig. 2(a). When the pulley input reverses its motion direction (to negative velocity), the motion at this pulley cannot immediately propagate to the pulley output, which exhibits some delays (points 1-2 in Fig. 2(b)). The friction force and the gap between the tendons and the sheaths prevent the immediate transmission of both motion and tension from the pulley input to the pulley output. As the friction on Sheath 01 decreases further, the pulley output begins to move- motion has been transmitted. When the friction in the Sheath 02 is further decreased, the two tendons in the Sheath 01 and Sheath 02 move together and the pulley output immediately follows the pulley input (see points 2-4 in the Fig. 2(b)). Similar characteristics are described for the reverse motion in clockwise direction of a pair of TSM. They are represented by points 4-5-6. It is further observed that the friction forces in the Sheath 01 and 02 are different due to the difference in tension transmission across the two tendons and pretension for ends of the tendons. Therefore, the total friction force $F$ is asymmetric for both negative and positive velocity. 


\section{A NEW FRICTION MODEL}

The normalized Bouc-Wen model, which is able to describe a wide range of shape of hysteresis behaviour, has been used in many applications. It has only five parameters to be identified instead of seven in comparison to the original Bouc-Wen model [27]. The model is described by a differential equation to capture the hysteresis phenomena of nonlinear system based on an internal state $\zeta$. It can be expressed by:

$$
\begin{gathered}
F=k_{x} x+k_{\zeta} \zeta \\
\dot{\zeta}=\rho\left(\dot{x}-\sigma|\dot{x}||\zeta|^{n-1} \zeta+(\sigma-1) \dot{x}|\zeta|^{n}\right)
\end{gathered}
$$

where $F$ is the total friction forces; $\rho>0, \sigma \geq 0, n \geq 1$ are constant coefficients that control the shapes and size of the hysteresis loops; $k_{x}>0, k_{\zeta}>0$ are factors that express the ratio of output displacement to the input displacement and the internal state, respectively; $x$ and $\dot{x}$ are the displacement and relative velocity at the proximal end respectively. The dot at the top of variables represents the first derivative with respect to time. The state variable $\zeta$ is the solution of differential Eq. (5).

The total friction forces, as shown in Fig. 2(b), in a pair of TSM are asymmetric for both positive and negative velocity. At near zero velocity, when the friction force changes from small displacement to large displacement (presliding to sliding regimes), an offset point exists on the hysteresis curves in the acceleration and deceleration directions. The model given by Eq. (4) and Eq. (5) is not able to capture the nonlinear friction and offset points of a pair of TSM. Hence, to describe the asymmetric characteristics, the main approach is to extend and modify the model given by Eq. (4) and Eq. (5) Two main modifications are carried out. The first is the modification of the relative velocity variable to be a function of the offset point and the acceleration of proximal end. The second is to extend the scale factor $k_{x}$ in Eq. (4) to become a smooth function of stiffness, velocity, and acceleration. The modified term $k_{x}(\dot{x}, \ddot{x})$, which controls the hysteresis asymmetric loops with large displacement for negative and positive velocity, is expressed by:

$$
\begin{aligned}
k_{x}(\dot{x}, \ddot{x}) & =0.5 k_{x}(1+\tanh (\dot{x})+(1-\tanh (\dot{x})) \operatorname{sign}(\mathrm{x}) \operatorname{sign}(\ddot{x})) \\
& =0.5 k_{x}\left(\frac{2 e^{2 \dot{x}}}{e^{2 \dot{x}}+1}+\left(\frac{2}{e^{2 \dot{x}}+1}\right) \operatorname{sign}(\mathrm{x}) \operatorname{sign}(\ddot{x})\right) \\
& =\frac{k_{x}}{e^{2 \dot{x}}+1}\left(e^{2 \dot{x}}+\operatorname{sign}(\mathrm{x}) \operatorname{sign}(\ddot{x})\right)
\end{aligned}
$$

where the hyperbolic tangent is defined as $\tanh (\dot{x})=\frac{e^{2 \dot{x}}-1}{e^{2 \dot{x}}+1}$

Therefore, the Eq. (4) and Eq. (5) are rewritten as:

$$
\begin{aligned}
& \dot{\zeta}=\rho\left(\dot{x}+\lambda \operatorname{sign}(\ddot{x})-\sigma|\dot{x}+\lambda \operatorname{sign}(\ddot{x})||\zeta|^{n-1} \zeta\right. \\
& \left.+(\sigma-1)(\dot{x}+\lambda \operatorname{sign}(\ddot{x}))|\zeta|^{n}\right)
\end{aligned}
$$

$$
F=k_{x}(\dot{x}, \ddot{x}) x+k_{\zeta} \zeta+v \dot{x}+F_{0}
$$

where $\lambda$ is the offset point of the adjustment; $\operatorname{sign}(\bullet)$ is the signum function that is defined by $\operatorname{sign}(\bullet)= \begin{cases}1 & \text { if }(\bullet)>0 \\ 0 & \text { if }(\bullet)=0 \\ -1 & \text { if }(\bullet)<0\end{cases}$

A viscous friction term is added to the Eq. (8) to capture the slope of the hysteresis loops for positive and negative velocity. $k_{x}$ is now the stiffness factor that controls the separate curves of the hysteresis loops; $v$ is the viscous coefficient that represents the incline line of the friction force in relation with the velocity. $F_{0}$ is an offset of friction force. A large value of $v$ means that the hysteresis loops has a steep slope.

The new model given by Eq. (7) and Eq. (8) represents the dynamic friction in a pair of TSM without any information of the tendon-sheath configurations in its structure. In addition, in the vicinity of zero velocity, it is able to capture the friction force unlike current models in the literature using the Coulomb model which has a discontinuity near zero velocity. Experimental demonstrations will be carried out in next sections.

\section{EXPERIMENTAL WORK AND IDENTIFICATION METHOD}

This section introduces the experimental work and identification algorithm to validate the proposed model approach.

\section{A. Experimental Work}

A pair of TSM is used to control each of the DOF in the robotic arms of the NOTES system shown in the Fig. 1. The two tendons are connected to a drive pulley (pulley 1) and a follower pulley (pulley 2). In order to increase the DOFs of the robotic arms in a very tight space of endoscope channels, the two TSMs are clustered into a flexible endoscope (see Fig. 2(a) and Fig. 3).

The schematic of the experimental setup is shown in Fig. 3. The TSMs are from Asahi Intecc Co. where the tendons have the size of WR $7 \times 7 \mathrm{D} 0.27 \mathrm{~mm}$ Teflon coated wire ropes outside and the sheaths are a round-wire coil with inner diameter of $0.36 \mathrm{~mm}$ and outer diameter of $0.8 \mathrm{~mm}$. The length of the two sheaths is $1.2 \mathrm{~m}$. The two TSMs are routed in a flexible tube, which models a flexible endoscope. FAULHABER 2642W024CR DC motors are used with the TSM. Actuator-Motor 01 is at the proximal end. ActuatorMotor 02 is used as a passive load or the environment load at the distal end of the system. Each of tendons is connected to the corresponding pulleys (Pulley 01 and 02 with the same diameter of 50mm) at the proximal and distal end as in Fig. 3, respectively. The two sheaths are fixed at relevant walls in order to prevent their sliding during the experiments. To measure the tension $T_{i}(i=1,2,3,4)$ at ends, four load cells LW-1020-50 from Interface Corporation are utilized. They are mounted on frictionless sliders, which are air bearings from NEW WAY air bearings. To measure the motion of the pulley, high resolution encoders E6D-CWZ1E 3600P/R 0.5M from OMRON are used. It is connected to the motor input (Encoder 01). During the experiment, the tendons are kept 
taut by pretensions. In addition, accumulated curve angles of flexible tube are maintained as constant. The feedback signals are evaluated using DS1104 controller from dSPACE and MATLAB Simulink from MathWorks.
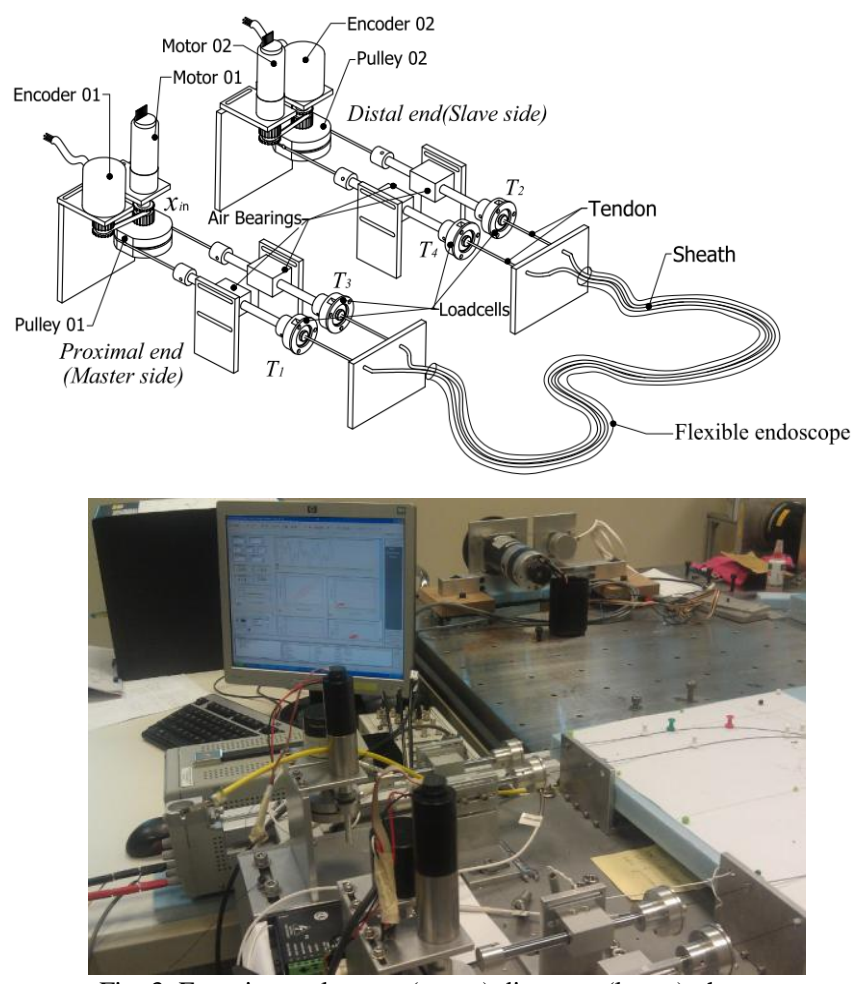

Fig. 3. Experimental setup: (upper) diagram; (lower) photo.

In the experimental works, various excitations are applied at the proximal end of the system. Recalling from Eq. (1) to Eq. (3), if the tensions at the proximal end and the distal end can be measured, the total friction forces in of a pair of TSM can be obtained. Fig. 4 shows the experimental results for the friction force under the excitation of a periodic motion (a combination of $1.05 \mathrm{rad}$ in amplitude and $0.2 \mathrm{~Hz}$ in frequency). The upper left panel shows the time history of excitation input (position) while the upper right panel depicts the time history of measured friction force from the experimental result. The relations between the friction force with velocity and displacement are introduced in the lower left and right panel of Fig. 4, respectively. It is observed that the friction forces for a pair of TSM follow a type of nonlinear hysteresis phenomena. The total friction forces are asymmetric for positive and negative velocity. There are separate hysteresis curves when the friction forces are accelerating and decelerating. In addition, at areas near zero velocity, there also are offset points of hysteresis curves in positive and negative velocity and acceleration. To show for more complex motion, non-periodic excitation signal (a combination of two signals: (1) amplitude of $0.52 \mathrm{rad}$ and frequency of $0.2 \mathrm{~Hz}$; (2) amplitude of $0.52 \mathrm{rad}$ and frequency of $0.25 \sqrt{3} \mathrm{~Hz}$ ) is also considered, as shown in Fig. 5. The upper left panel shows the time history of displacement applied to the proximal pulley. The upper right panel depicts the time history of the total friction forces between the tendons and the sheaths. The relations of friction with velocity and displacement are introduced in the lower left and lower right panel of this figure, respectively. It is observed that the friction behavior is quite complex in the case of non-periodic motion. These nonlinearities will be characterized by the proposed model and will be discussed in the next sections.
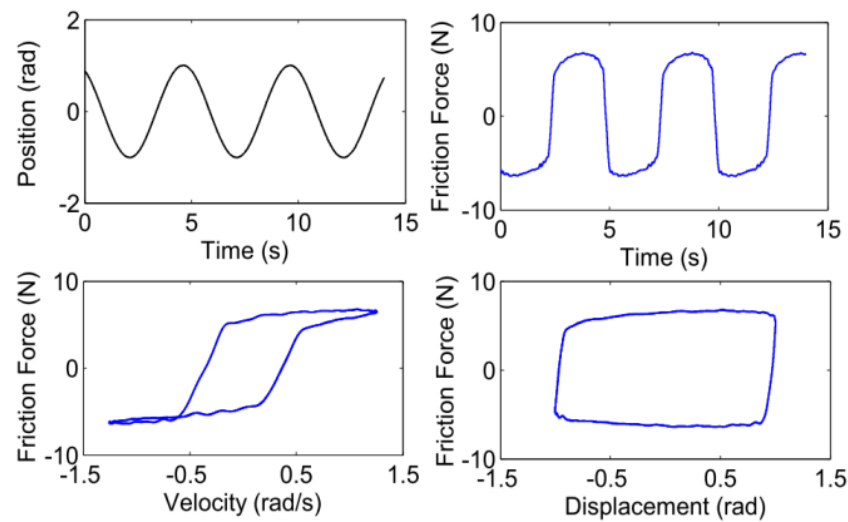

Fig. 4. Experimental results for a single periodic motion (a combination of $1.05 \mathrm{rad}$ in amplitude and $0.2 \mathrm{~Hz}$ in frequency).
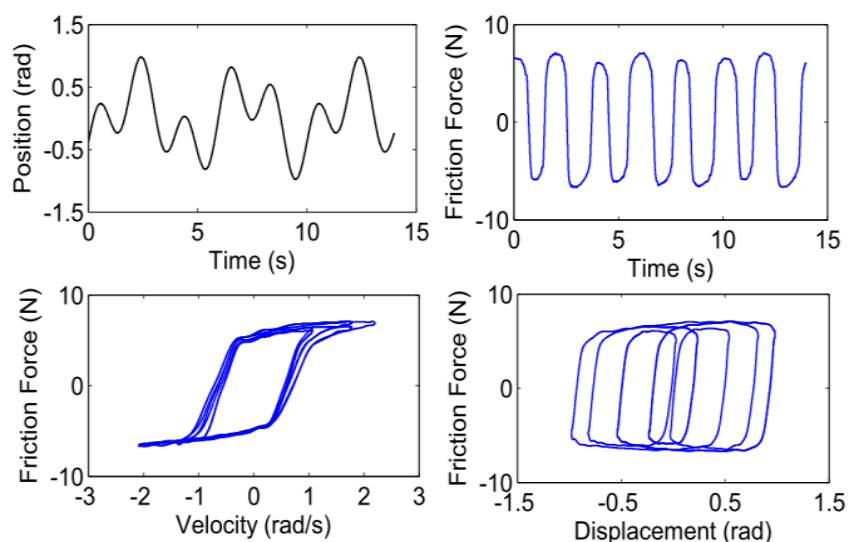

Fig. 5. Experimental results for multiple periodic motions (a combination of two signals: (1) amplitude of $0.52 \mathrm{rad}$ and frequency of $0.2 \mathrm{~Hz}$; (2) amplitude of $0.52 \mathrm{rad}$ and frequency of $0.25 \sqrt{3} \mathrm{~Hz}$ ).

\section{B. Identification Algorithm}

To validate the proposed friction and backlash hysteresis models, parameters of the models are firstly identified using Genetic Algorithm (GA) [29]. The GA will generate an initial guess of model parameters. Subsequently, the obtained model parameters will be used as an initial guess in the Nelder-Mead Simplex method to refine the result. During the identification process, MATLAB identification Toolbox from MathWorks is utilized to carry out the work. To evaluate the performance of the identification, the mean square error $(M S E)$ is used and expressed by:

$$
M S E=\frac{1}{N} \sum_{i=1}^{N}\left(f_{\text {actual }}(i)-f_{\text {model }}(i)\right)^{2}
$$

Subject to suitable constraints; $i$ is the sampling index and $N$ is total number of samples from experimental results, $f_{\text {actual }}$ is actual experimental data of the tendon-sheath system (displacement or friction force), $f_{\text {model }}$ is the estimated values obtained from the proposed friction and backlash hysteresis models. The fitness function MSE is defined as the mean square error between the proposed models and actual experimental data. All signals are filtered using zero-phase digital filtering with filtfilt command in MATLAB. 


\section{IDENTIFICATION RESULTS AND VALIDATIONS}

To validate the proposed friction model given by Eq. (6) to Eq. (8), a set of model parameters $\rho, \lambda, \sigma, n, k_{\zeta}, v$ have to be identified. In this validation, periodic motion (a a combination of amplitude of $1.05 \mathrm{rad}$ and frequency of $0.2 \mathrm{~Hz}$ ) and non-periodic motion (a combination of two signals: (1) amplitude of $0.52 \mathrm{rad}$ and frequency of $0.2 \mathrm{~Hz}$; (2) amplitude of $0.52 \mathrm{rad}$ and frequency of $0.25 \sqrt{3} \mathrm{~Hz}$ ) are considered at the proximal of the system. Fig. 4 shows the identification results for the estimated friction model using a periodic motion of input signal. The model parameter, which are optimized using algorithm in section 4.2, are given as $\rho=26.6659, \lambda=-0.1172, \sigma=1.7512, n=2.0458, k_{x}=10.121$ $, k_{\zeta}=127.9493, v=25.8085, F_{0}=0.2555$.

The comparison between the proposed model and real experimental data in comparison with time history is presented in the upper left panel of Fig. 6. It is shown that the proposed model given by Eq. (6) to Eq. (8) can accurately predict the real friction force for a pair of TSM without using any tendon-sheath configuration information (with the mean square error (MSE) is 0.0259$)$. The time history of error data is also illustrated in this figure. The upper right panel of Fig. 6 depicts the results of the estimated friction model versus velocity. It is observed that the friction force in the small and large displacement is well captured and it is smooth. In addition, it is able to track the separate hysteresis loops in the sliding regime (large displacement). The relation between the friction force and displacement is presented in the lower panel of Fig. 6.

As mentioned in Section I, haptic feedback plays an important role in safe surgery. Without haptic feedback, the surgeon is not able to feel as if they have directly touched or make contact with the real tissue. Hence, the ultimate goal of this paper is to provide the force information to surgeon (torque output- $T_{\text {out }}$ ) via haptic devices. Therefore, the output torque is directly estimated based on the torque input $T_{i n}$ and the proposed friction model (see Eq. (3)). The time history of estimated the input and output torque from the proposed model and the measured data can be seen in the lower right panel of Fig. 6.
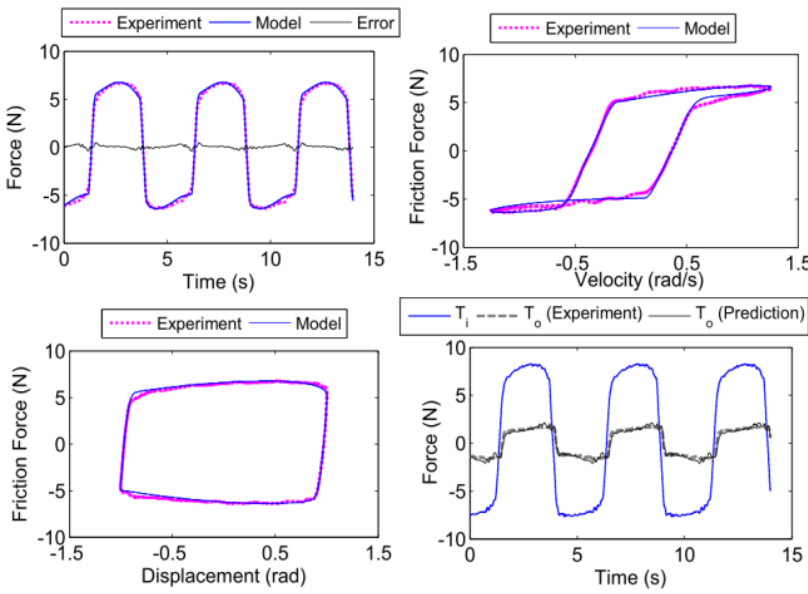

Fig. 6. Identification results for a single periodic motion.

The capability of the proposed friction model is also demonstrated in the case of more complex motion (non-periodic motion-a combination of two signals: (1) amplitude of $0.52 \mathrm{rad}$ and frequency of $0.2 \mathrm{~Hz}$; (2) amplitude of $0.52 \mathrm{rad}$ and frequency of $0.25 \sqrt{3} \mathrm{~Hz}$ ). Fig. 7 illustrates identification and comparison results. The upper left panel of this figure introduces the time history of identification result between the proposed model and experimental data. The corresponding error is also presented in this figure. It is shown that the characteristics of the nonlinear friction model are confirmed by a good agreement between the two data (estimation and real experiment). For better comparison and observation, the mean square error is also given in this case, i.e. $M S E=0.0293$. The relations between the friction model (estimation and experiment) with velocity and displacement are illustrated in the upper right and lower left panels of Fig. 7, respectively. From those figures, it is confirmed that the new friction model can well track the friction force for a pair of TSM, especially in the large displacement where the hysteresis loops are asymmetric as well as the smoothness of friction curves are guaranteed. Finally, to provide the force information to haptic devices and subsequently to surgeon, the estimated torque and real experimental data of the output (distal end) are introduced in the lower left panel of Fig. 7. From these result, it can be seen that the proposed model approach does not only track the periodic motion of signal but also well follows the complex motion like non-periodic motion.
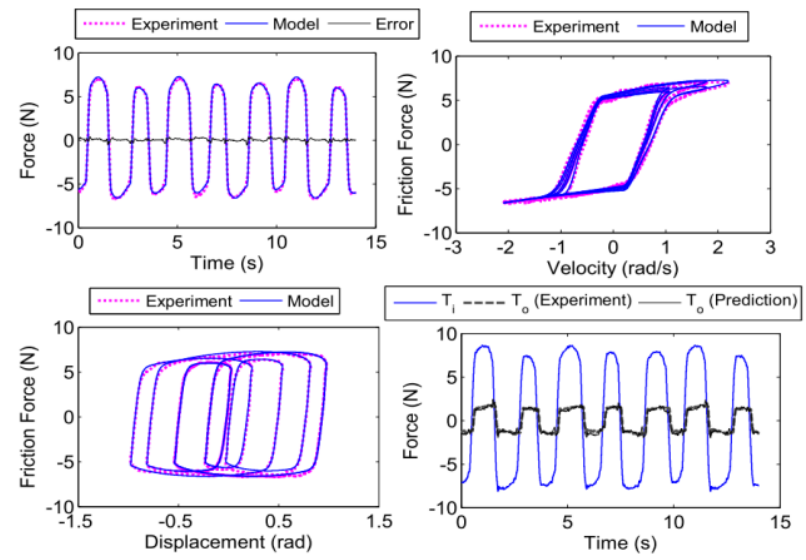

Fig. 7. Identification results for multiple periodic motions.

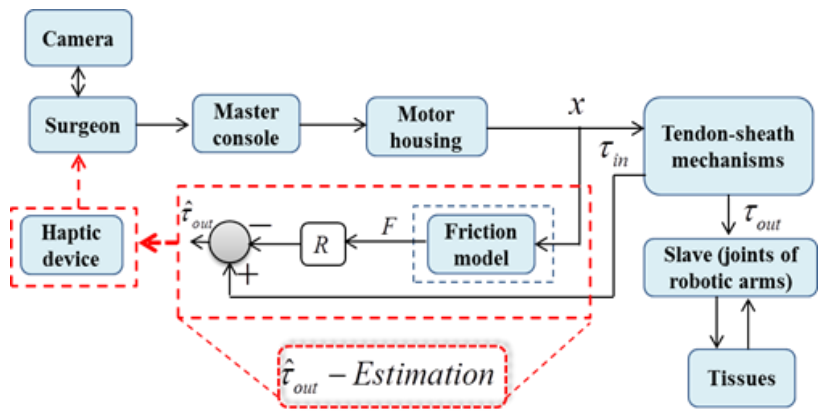

Fig. 8. Diagram of estimated force structure.

The overview of a NOTES system has been given in Fig. 1. To provide the torque information at distal end of robotic arms, an estimated force structure is shown in Fig. 8. Under the lack of feedback at the distal end, mathematical model given by Eq. 6 to Eq. 8 is used to provide force information to haptic device. The estimated values in the proposed structure only use the force information and position at proximal end of 
the NOTES system without utilizing any feedback from the distal end. The surgeon controls the slave manipulator (possess robotic arms) using the master console to perform the surgical tasks. Torque information from sensors is externally provided. Having the position and torque information at proximal end, the output torque at distal end can be accurately estimated, as introduced in Fig. 6 and Fig. 7 as well as in the structure of the Fig. 8.

\section{CONCLUSION}

Nonlinear friction causes the tension losses in the TSM. At microscopic displacement, the friction force rapidly changes and results in difficult estimation. In addition, the nonlinear characteristics in the large displacement is quite complex in the case of TSM. It is asymmetric for positive and negative velocity. For acceleration and deceleration directions, there are separate branches for hysteresis curves. This paper has introduced a new dynamic friction model to solve the haptic feedback problems for flexible endoscopic system using a pair of tendon-sheath mechanism. The proposed model, which uses a modification of the normalized Bouc-Wen model and acceleration information, is able to capture the asymmetric friction force for small and large displacements and it is smooth. In comparison with current approaches in the literatures where a discontinuous model like Coulomb is utilized, our proposed model guarantees a smooth estimation of friction force for small and large displacements. In addition, the proposed model can give a smooth transition between the two stages (sliding regime-small displacement and presliding regime-large displacement) and is capable to predict the nonlinear friction force vicinity of zero velocity where the system is stationary, regardless the sheath configuration in the model structure.

Validation experiments have been carried out to evaluate the proposed model. An efficient identification method (Genetic Algorithm) has firstly used to generate a rough initial guess of model parameters. The obtained results have been refined using the Nelder-Mead Simplex Method. The comparisons between the proposed model and the experiment data have shown a good agreement for the approach. A diagram of estimated force has been given to provide force information to haptic devices and then to the surgeon under the availability of the force and position information at proximal end. It can be concluded that the proposed model promises an efficient approach not only in accurately predicting the force for haptic feedback but also in any surgical devices that have similar friction characteristics as the tendon sheath.

Future activities will be conducted by developing the control compensation scheme for the TSM using in NOTES system. Haptic devices will be utilized to provide real force information to the surgeon during operation. A hysteresis compensation for both position and friction force feedback will be also developed using suitable nonlinear control schemes and taking into consideration for higher degrees of freedom in the system. Subsequently, real time experimental works on animals will be carried out using the proposed model.

\section{REFERENCES}

[1] L. Ott, F. Nageotte, P. Zanne, and M. de Mathelin, "Robotic assistance to flexible endoscopy by physiological-motion tracking," IEEE Transactions on Robotics, vol. 27, no. 2, pp. 346-359, 2011.

[2] Y. Zhou, H. Ren, M. Q. H. Meng, Z. T. H. Tse, and H. Yu, "Robotics in natural orifice transluminal endoscopic surgerys," Journal of Mechanics in Medicine and Biology, vol. 13, no. 2, pp. 1-11, 2013.

[3] R. Förster, M. Storck, J. Schäfer, E. Hönig, G. Lang, and F. Liewald, "Thoracoscopy versus thoracotomy: a prospective comparison of trauma and quality of life," Langenbeck's Arch Surg, vol. 387, no. 1, pp. 32-36, 2002.

[4] I. Nagahiro, A. Andou, M. Aoe, Y. Sano, H. Date, and N. Shimizu, "Pulmonary function, postoperative pain, and serum cytokine level after lobectomy: a comparison of VATS and conventional procedure,' The Annals of Thoracic Surgery, vol. 72, no. 2, pp. 362-365, 2001.

[5] J. Bodner, H. Wykypiel, G. Wetscher, and T. Schmid, "First experiences with the da Vinci ${ }^{\mathrm{TM}}$ operating robot in thoracic surgery," European Journal of Cardio-Thoracic Surgery, vol. 25, no. 5, pp. 844-851, 2004.

[6] V. Hayward, O. R. Astley, M. Cruz-Hernandez, D. Grant, and G. Robles-De-La-Torre, "Haptic interfaces and devices," Sensor Review, vol. 24, no. 1, pp. 16-29, 2004

[7] A. M. Okamura, L. N. Verner, C. E. Reiley, and M. Mahvash, "Haptics for robot-assisted minimally invasive surgery," in Robotics Research, M. Kaneko and Y. Nakamura, Eds., Springer Berlin Heidelberg, 2011, pp. 361-372.

[8] A. M. Okamura, "Haptic feedback in robot-assisted minimally invasive surgery," Current Opinion in Urology, vol. 19, no. 1, pp. 102-107, 2009.

[9] Y. Kobayashi, P. Moreira, C. Liu, P. Poignet, N. Zemiti, and M. G. Fujie, "Haptic feedback control in medical robots through fractional viscoelastic tissue model," in Proc. Annual International Conference on Engineering in Medicine and Biology Society, 2011, pp. 6704-6708.

[10] A. E. Quaid and R. A. Abovitz, "Haptic information displays for computer-assisted surgery," in Proc. IEEE International Conference on Robotics and Automation, 2002, pp. 2092-2097.

[11] M. Kaneko, T. Yamashita, and K. Tanie, "Basic considerations on transmission characteristics for tendon drive robots," in Proc. Fifth International Conference on Advanced Robotics, 'Robots in Unstructured Environments', Pisa, Italy, 1991, pp. 827-832

[12] M. Kaneko, M. Wada, H. Maekawa, and K. Tanie, "A new consideration on tendon-tension control system of robot hands," in Proc. IEEE International Conference on Robotics and Automation, 1991, pp. 1028-1033.

[13] M. Kaneko, W. Paetsch, and H. Tolle, "Input-dependent stability of joint torque control of tendon-driven robot hands," IEEE Transactions on Industrial Electronics, vol. 39, no. 2, pp. 96-104, 1992.

[14] T. N. Do, T. Tjahjowidodo, M. W. S. Lau, T. Yamamoto, and S. J. Phee, "Hysteresis modelling and position control of tendon-sheath mechanism in flexible endoscopic systems," Mechatronics, vol. 24, no. 1, pp. 12-24, 2014.

[15] G. Palli and C. Melchiorri, "Model and control of tendon-sheath transmission systems," in Proc. IEEE International Conference on Robotics and Automation, Orlando, FL, 2006, pp. 988-993.

[16] G. Palli and C. Melchiorri, "Optimal control of tendon-sheath transmission systems," in Proc. the 8th International IFAC Symposium on Robot Control, Bologna, Italy, 2006, pp. 73-78.

[17] F. Tian and X. Wang, "The design of a tendon-sheath-driven robot," in Proc. 15th International Conference on Mechatronics and Machine Vision in Practice, 2008, pp. 280-284.

[18] L. Chen and X. Wang, "Modeling of the tendon-sheath actuation system," in Proc. 19th International Conference on Mechatronics and Machine Vision in Practice, Auckland, 2012, pp. 489-494.

[19] S. C. Low, S. J. Phee, P. Valdastri, A. Menciassi, and P. Dario, "Tendon sheath analysis for estimation of distal end force and elongation," in Proc. IEEE/ASME International Conference on Advanced Intelligent Mechatronics, 2009, pp. 332-337.

[20] V. Agrawal, W. J. Peine, and B. Yao, "Modeling of transmission characteristics across a cable-conduit system," IEEE Transactions on Robotics, vol. 26, no. 5, pp. 914-924, 2010.

[21] V. Agrawal, W. J. Peine, and B. Yao, "Modeling of a closed loop cable-conduit transmission system," in Proc. IEEE International Conference on Robotics and Automation, Pasadena, CA, 2008, pp. 3407-3412.

[22] T. Tjahjowidodo, F. Al-Bender, H. V. Brussel, and W. Symens, "Friction characterization and compensation in electro-mechanical 
systems," Journal of Sound and Vibration, vol. 308, no. 3-5, pp. 632-646, 2007

[23] C. C. de Wit, H. Olsson, K. J. Astrom, and P. Lischinsky, "A new model for control of systems with friction," IEEE Transactions on Automatic Control, vol. 40, no. 3, pp. 419-425, 1995.

[24] T. N. Do, T. Tjahjowidodo, M. W. S. Lau, and S. J. Phee, "Nonlinear modeling and parameter identification of dynamic friction model in tendon sheath for flexible endoscopic systems," in Proc. the 10th International Conference on Informatics in Control, Automation and Robotics,Reykjavik, Iceland, 2013, vol. 2, pp. 5-10.

[25] T. N. Do, T. Tjahjowidodo, M. W. S. Lau, and S. J. Phee, "An investigation of friction-based tendon sheath model appropriate for control purposes," Mechanical Systems and Signal Processing, vol. 42 no. 1, pp. 97-114, 2013.

[26] T. N. Do, T. Tjahjowidodo, M. W. S. Lau, and S. J. Phee, "Dynamic friction model for tendon-sheath actuated surgical robots: modelling and stability analysis," in Proc. the 3rd IFToMM International Symposium on Robotics and Mechatronics, Singapore, 2013, pp. 302-311.

[27] F. Ikhouane and J. Rodellar, Systems with Hysteresis: Analysis, Identification and Control Using the Bouc-Wen Model, Hoboken John Wiley \& Sons, Ltd., 2007.

[28] F. Ikhouane, J. Hurtado, and J. Rodellar, "Variation of the hysteresis loop with the bouc-wen model parameters," Nonlinear Dynamics, vol. 48, no. 4, pp. 361-380, 2007.

[29] M. Mitchell, Introduction to Genetic Algorithms, MIT Press, 1996.

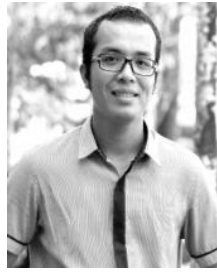

Thanh Nho Do received the B. Eng. degree in mechanical engineering from Ho Chi Minh City University of Technology, Vietnam. He is currently a $\mathrm{PhD}$ candidate in division of mechatronics and Design, school of MAE, NTU, Singapore. His research interests include developing nonlinear dynamic friction, backlash hysteresis models, and nonlinear and adaptive control with uncertain parameters in medical devices and flexible systems.

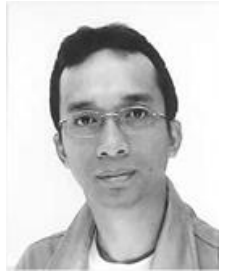

Tegoeh Tjahjowidodo received the Ph.D. degree from the Katholieke Universiteit Leuven, Heverlee, Belgium, in 2006. He was joining the Flanders' Mechatronics Technology Center, Belgium, a research center in the mechatronics area, where he was involved in several projects, mainly in nonlinear dynamics. He is currently an assistant professor at the School of Mechanical and Aerospace Engineering, Nanyang Technological University, Singapore. His main research interests are in nonlinear dynamics, modeling, identification and control.

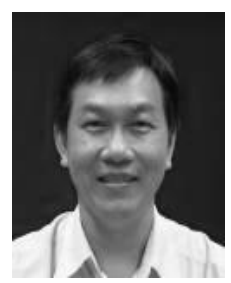

Michael Wai Shing Lau is currently with Newcastle University, UK. His primary interests are in mechatronics and control of mechanical systems. His research activities include motion control, active vibration control, and design and control of mobile robotics including underwater vehicles.

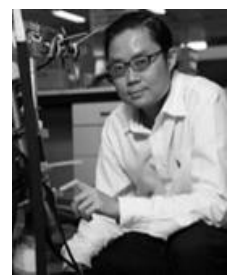

Soo Jay Phee received M. Eng. degree from Nanyang Technological University Singapore 1999. In 2002, he received the Ph.D. degree from the Scuola Su-periore SantAnna, Pisa, Italy, under a European Union scholarship. He is currently an associate professor at NTU. He is the principal investigator of some projects. His research interests include medical robotics and mechatronics in medicine 